\title{
Progress toward small-scale field trials of coastal enhanced weathering of olivine
}

\author{
CHEYENNE MOREAU ${ }^{1}$, FRANCESC MONTSERRAT ${ }^{2}$, \\ TOM GREEN $^{1}$, ERIC MATZNER ${ }^{1}$, DREW SYVERSON ${ }^{1}$, \\ OLIVIER SULPIS ${ }^{3}$, MATT HAYDEN ${ }^{1}$, PALOMA LOPEZ ${ }^{1}$ \\ AND STEPHEN J ROMANIELLO ${ }^{4}$ \\ ${ }^{1}$ Project Vesta \\ ${ }^{2}$ University of Amsterdam \\ ${ }^{3}$ Department of Earth Sciences \\ ${ }^{4}$ University of Tennessee, Knoxville \\ Presenting Author: cheyenne@projectvesta.org
}

Enhanced weathering has promising carbon uptake potential, but significant uncertainty around environmental impacts, cobenefits, and general feasibility. In order to consider large-scale intervention using any carbon sequestration strategy, resolving these uncertainties is necessary and urgent. Coastal enhanced weathering of olivine (CEWO) places mined and then ground olivine-rich ultramafic rocks (such as dunite) into coastal areas like beaches, and leverages the mechanical action of wave energy to accelerate the weathering process. Grinding olivine is costly (in dollars and carbon) and also essential for increasing surface area reactivity, so CEWO can reduce costs substantially. This approach can also be incorporated into planned beach nourishment and coastal development projects to facilitate widespread use.

By-products including nickel, chromium, and silicon are released during the olivine dissolution process. Given the potential toxicity of metals, and fertilization effects of increased dissolved silica in seawater, it is critical to understand the fate and ecological effects of olivine weathering, in both controlled and natural settings. We present the results of laboratory toxicity experiments following standard United States Environmental Protection Agency (USEPA) protocol for assessing acute, chronic, and bioaccumulation toxicity in a set of model organisms that represent a diversity of life histories and trophic levels. We also discuss preliminary laboratory results on diatom fertilization potential with CEWO, as well as other potential effects on organisms like physical smothering by the olivine sand layer, and the effects of olivine particles on bioturbators' digestive systems.

We introduce our proposed research program that includes lab experiments on dissolution kinetics and the effects of olivine on growth rates of calcifying organisms, followed by mesocosm studies that investigate indirect effects in a model community. The first CEWO field trial is being planned in the Carribean with geochemical and ecological monitoring, in order to 1.) determine the ecological effects of placing olivine sand in a marine environment, and 2.) assess whether CEWO produces alkalinity and other measurable geochemical changes indicating the removal of atmospheric CO2. Project Vesta, a philanthropicallyfunded non-profit, is conducting this research to advance our understanding of CEWO and its potential uses and limitations. 